\title{
Mass Hierarchy via Mössbauer and Reactor Neutrinos
}

\author{
Stephen J. Parke, ${ }^{\text {a* }}$ H. Minakata,${ }^{\mathrm{b}}$ H. Nunokawa, ${ }^{\mathrm{c}}$ R. Zukanovich Funchal ${ }^{\mathrm{d}}$ \\ aTheoretical Physics Dept., Fermi National Accelerator Laboratory, Batavia, IL 60510, USA \\ ${ }^{\mathrm{b}}$ Department of Physics, Tokyo Metropolitan University, Hachioji, Tokyo 192-0397, Japan \\ ' Dept. de Física, Pontifícia U. Católica do Rio de Janeiro, C. P. 38071, 22452-970, Rio de Janeiro, Brazil \\ ${ }^{\text {d} I n s t i t u t o ~ d e ~ F i ́ s i c a, ~ U n i v e r s i d a d e ~ d e ~ S a ̃ o ~ P a u l o, ~ C . ~ P . ~ 66.318, ~ 05315-970 ~ S a ̃ o ~ P a u l o, ~ B r a z i l ~}$
}

We show how one could determine the neutrino mass hierarchy with Mössbauer neutrinos and also revisit the question of whether the hierarchy can be determined with reactor neutrinos.

\section{Mössbauer Neutrinos}

The term Mössbauer Neutrinos refers to a source-detector combination where there is no recoil in the production and absorption of the neutrino so that the neutrinos have resonant energy thereby enhancing the capture cross section by approximately 10 orders of magnitude!

$$
\text { Source : }{ }^{3} \mathrm{H} \rightarrow\left({ }^{3} \mathrm{He}+e^{-}\right)_{B}+\bar{\nu}_{e}
$$$$
\text { Detector : } \quad \bar{\nu}_{e}+\left({ }^{3} \mathrm{He}+e^{-}\right)_{B} \rightarrow{ }^{3} H
$$

Such a source-detector combination would be wonderful for neutrino physics if it could be practically realized, see [1. Here we address the question of whether such a system could be used to determine the neutrino mass hierarchy.

\section{2. $\nu_{e}$ Disappearance Probability}

The vacuum $\nu_{e}$ survival probability using $\Delta_{i j} \equiv \Delta m_{i j}^{2} L / 4 E\left(\Delta m_{i j}^{2} \equiv m_{i}^{2}-m_{j}^{2}\right)$ as shorthand for the kinematical phase, can be written without any approximation as

$$
\begin{array}{r}
P\left(\nu_{e} \rightarrow \nu_{e}\right)=1-\cos ^{4} \theta_{13} \sin ^{2} 2 \theta_{12} \sin ^{2} \Delta_{21} \\
-\sin ^{2} 2 \theta_{13}\left[\cos ^{2} \theta_{12} \sin ^{2} \Delta_{31}+\sin ^{2} \theta_{12} \sin ^{2} \Delta_{32}\right] .
\end{array}
$$

The first term gives the contribution from the solar $\Delta m^{2}$ whereas the last two terms are the contributions from the two atmospheric $\Delta m^{2}$ s. This

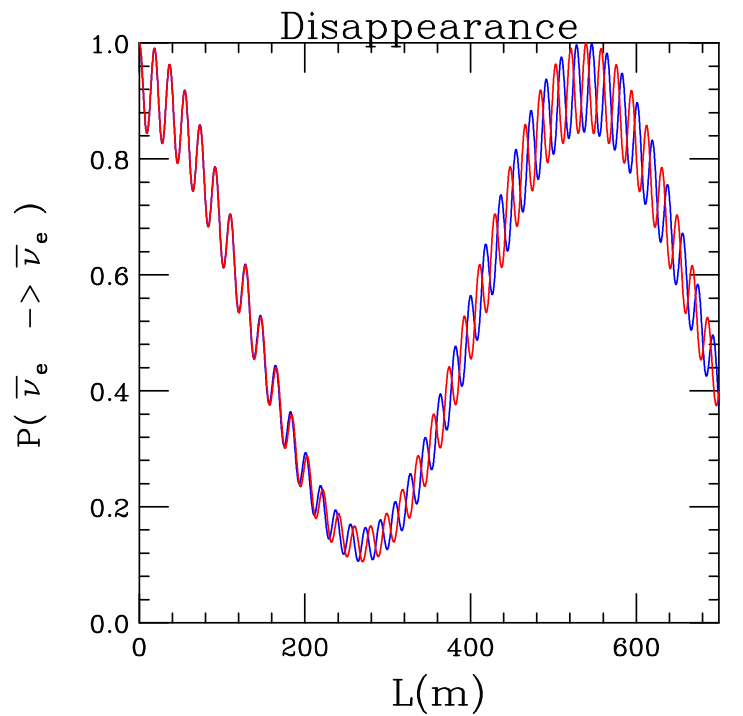

Figure 1. The antineutrino survival probability $P\left(\bar{\nu}_{e} \rightarrow \bar{\nu}_{e}\right)$ is plotted as a function of $L$ for the $18.6 \mathrm{keV}$ Mössbauer Neutrinos for both the normal (blue) and inverted (red) hierarchies. The advancement/retardation in the phase of the atmospheric oscillation is clearly visible beyond the first solar oscillation minimum. Here the quantity $\Delta m_{e e}^{2} \equiv c_{12}^{2}\left|\Delta m_{31}^{2}\right|+s_{12}^{2}\left|\Delta m_{32}^{2}\right|$ is the same for both hierarchies.

*Presenter. email:parke@fnal.gov 


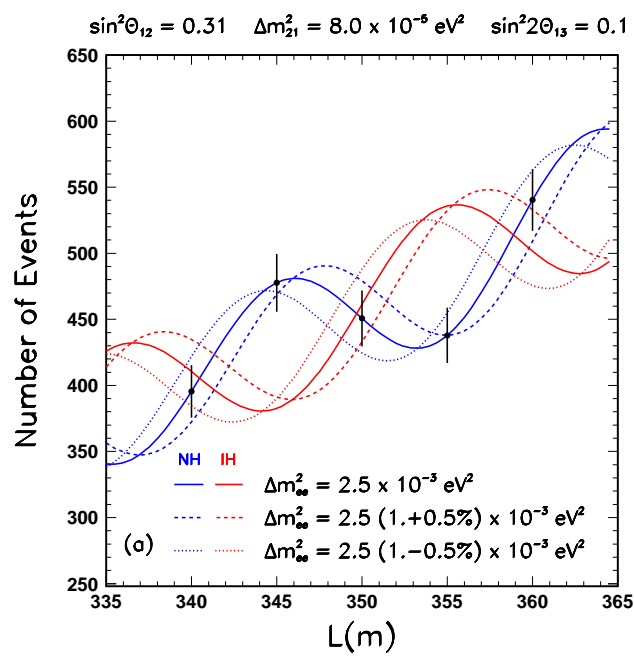

Figure 2. Plotted are the expected number of events to be collected by detectors placed at the distances $340,345,350,355$ and $360 \mathrm{~m}$ from the source for $\Delta m_{\mathrm{ee}}^{2}=2.5 \times 10^{-3} \mathrm{eV}^{2}$ and $\sin ^{2} 2 \theta_{13}=$ 0.1 for the normal hierarchy, indicated by solid circles with error bars.

atmospheric contribution consists of two waves with slightly different frequencies which leads to

- a modulation of the amplitude of the atmospheric oscillations by the factor $\sqrt{1-\sin ^{2} 2 \theta_{12} \sin ^{2} \Delta_{21}}$

- and an advancement (retardation) of the phase of the atmospheric oscillation by $2 \pi \sin ^{2} \theta_{12}$ for every solar oscillation $\left(\Delta_{21} \rightarrow \Delta_{21}+\pi\right)$ assuming the normal (inverted) hierarchy.

The easiest way to see these two effects is to combine the two atmospheric oscillation terms into a single term as follows:

$$
\left.\begin{array}{c}
2\left[\cos ^{2} \theta_{12} \sin ^{2} \Delta_{31}+\sin ^{2} \theta_{12} \sin ^{2} \Delta_{32}\right]= \\
{\left[1-\sqrt{1-\sin ^{2} 2 \theta_{12} \sin ^{2} \Delta_{21}} \cos \left(2 \Delta_{\text {ee }} \pm \phi_{\odot}\right)\right.}
\end{array}\right]
$$

where the argument of the new cosine term, $\left(2 \Delta_{\mathrm{ee}} \pm \phi_{\odot}\right)$, has been separated into a linear term in $\mathrm{L} / \mathrm{E}, 2 \Delta_{e e} \equiv \Delta m_{e e}^{2} L / 2 E$, and the remainder, $\pm \phi_{\odot}$, whose derivative wrt $\mathrm{L} / \mathrm{E}$ vanish at $\mathrm{L} / \mathrm{E}=0$.

The $\Delta m^{2}$ associated with the linear term, $2 \Delta_{\text {ee }}$, is given by

$$
\begin{aligned}
\Delta m_{e e}^{2} & \equiv c_{12}^{2}\left|\Delta m_{31}^{2}\right|+s_{12}^{2}\left|\Delta m_{32}^{2}\right| \\
& =\left|m_{3}^{2}-\left(c_{12}^{2} m_{1}^{2}+s_{12}^{2} m_{2}^{2}\right)\right|
\end{aligned}
$$

and is the electron flavor weighted average of $\left|\Delta m_{31}^{2}\right|$ and $\left|\Delta m_{32}^{2}\right| . \Delta m_{e e}^{2}$ is the atmospheric $\Delta m^{2}$ measured by a electron neutrino disappearance experiment in the first few oscillations from the source.

The $\pm \phi_{\odot}$ contains everything else, with the positive (negative) sign for the normal (inverted) hierarchies, and only depends on the solar $\Delta m^{2}$ as follows

$\phi_{\odot} \equiv \arctan \left(\cos 2 \theta_{12} \tan \Delta_{21}\right)-\Delta_{21} \cos 2 \theta_{12}$.

$\phi_{\odot}$ is a monotonically increasing function of $\Delta_{21}$ and changes by $2 \pi \sin ^{2} \theta_{12}$ for every $\pi$ that $\Delta_{21}$ increases, i.e. $\phi_{\odot}\left(\Delta_{21}+\pi\right)=\phi_{\odot}\left(\Delta_{21}\right)+2 \pi \sin ^{2} \theta_{12}$.

It is this advancement or retardation of the phase of the atmospheric oscillation which can exploited to determine the mass hierarchy using only the $\nu_{e}$ vacuum disappearance channel. Thus, the strategy is as follows: to make a precise determination of the atmospheric $\Delta m^{2}$ near the first few atmospheric oscillation and then to go out beyond the first solar minimum and determine the phase of the atmospheric oscillation at the point where the atmospheric oscillations from normal and inverted hierarchy scenarios are close to 180 degrees out of phase, see Figures 1 and 2 Since this point is 20 atmospheric oscillation from the source the precise determination of the $\Delta m^{2}$ (better than $1 \%$ ) near the source is required so that one can determine whether the measured phase is associated with the normal or inverted hierarchy. Also the $\mathrm{L} / \mathrm{E}$ of the events is required to be determined by better than $1 \%$ or otherwise the atmospheric oscillations are averaged out. For an ultra-monochromatic neutrino source like Mössbauer Neutrinos this is not a very stringent requirement provided that the source and detector are relatively compact. Figure 3 gives the sensitivity to determining the mass hierarchy assuming a certain number of events per detector as discussed in [2]. 


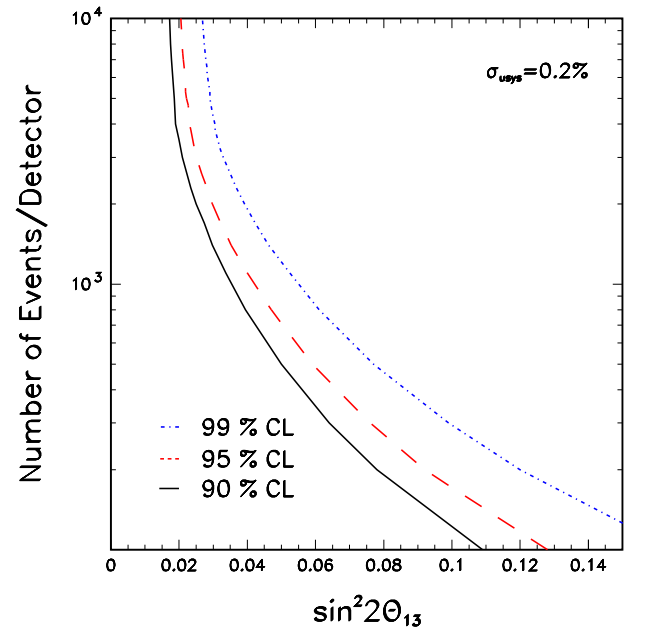

Figure 3. The region of sensitivity to resolving the mass hierarchy in $\sin ^{2} 2 \theta_{13}$-event number (per detector) space. The black solid, the red dashed, and the blue dotted curves denote the region boundary at $90 \%, 95 \%$, and $99 \%$ CL, respectively. The uncorrelated systematic uncertainties are assumed to be of $0.2 \%$.

We now turn to the question of whether reactor neutrinos can be used to determine the neutrino mass hierarchy using the difference in the disappearance probability for the normal and inverted hierarchies. This issue has been discussed in some detail in a recent paper with respect to the Hanohano experiment, see [3. In Fig. 4 we have plotted the percentage difference in the disappearance probability assuming

$\Delta m_{e e}^{2}(I H)=1.008 \times \Delta m_{e e}^{2}(N H)$

with this choice the difference between the two hierarchies is minimized in the energy window 2$8 \mathrm{MeV}$ accessible with reactors. If we know the energy of the neutrinos exactly, $E_{\text {obs }}=E_{\text {true }}$, then the difference between the two hierarchies is approximately $1 \%$.

However, if the measured neutrino energy differs from the true energy by a small amount, say

$E_{\text {obs }}=1.015 E_{\text {true }}-0.07 \mathrm{MeV}$,

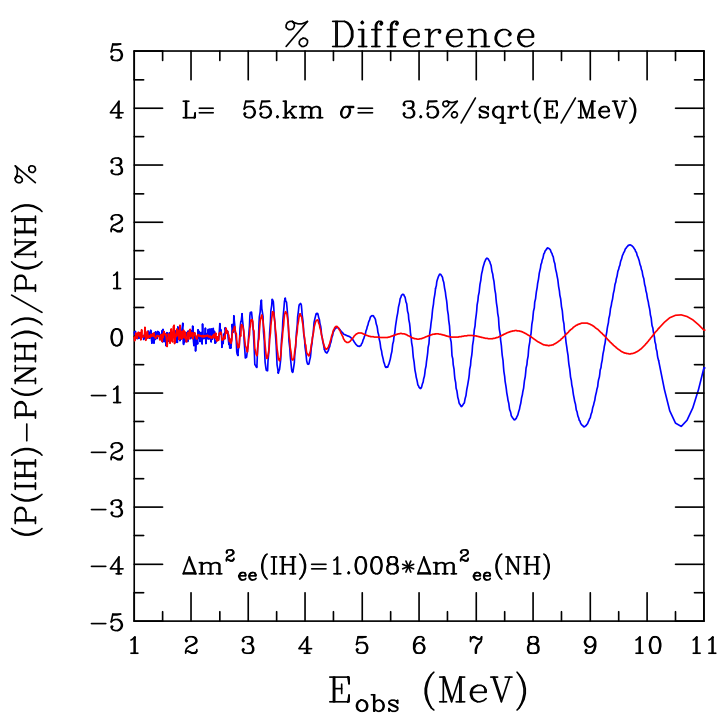

Figure 4. The percentage difference between the inverted hierarchy and the normal hierarchy. The blue curve is assuming $E_{o b s}=E_{\text {true }}$ and maximum difference is less than $2 \%$. Whereas for the red curve we have assumed that $E_{o b s}=$ $1.015 E_{\text {true }}-0.07 \mathrm{MeV}$ for the $\mathrm{IH}$, so as to represent a relative calibration uncertainty in the neutrino energy. Here the maximum percentage difference is less than $0.5 \%$.

then the difference between the inverted hierarchy oscillation probability using $E_{o b s}$ and the normal hierarchy with $E_{\text {true }}$ can be considerable smaller than $1 \%$. Thus, the requirements for determining the neutrino mass hierarchy with reactor neutrinos are very stringent.

I wish to thank the organizers of NOW 2008, Prof. Fogli and Prof. Lisi, for a wonderfully stimulating atmosphere.

\section{REFERENCES}

1. R. S. Raghavan, arXiv:hep-ph/0601079.

2. H. Minakata, H. Nunokawa, S. J. Parke and R. Zukanovich Funchal, Phys. Rev. D 76, 053004 (2007) arXiv:hep-ph/0701151.

3. J. Learned, S. T. Dye, S. Pakvasa and R. C. Svoboda, Phys. Rev. D 78, 071302 (2008) arXiv:hep-ex/0612022. 